the medium itself as a major tool in psychiatric research.

An extensive bibliography is appended. It is somewhat inaccurate in that certain authors cited in the main body of Onder's work do not appear in the bibliography. The absence of an index makes the book less useful than it might be otherwise. However, it is certainly a valuable addition to any psychiatric department library where television is used and is a must for all health science libraries.-Brigitte L. Kenney, University of Mississippi Medical Center.

Agricultural Sciences Information Network Development Plan: EDUCOM Research Report No. 169. Boston, 1969. 74p. $\$ 6.00$.

At the behest of the National Agricultural Library and in accordance with policy guidelines established by an advisory committee representing users of agricultural information, an EDUCOM study team set about developing a network concept and implementation plan. The specific goal which emerged was to devise a "program for mobilizing our proliferating agricultural and biological information resources and sharing them with the landgrant colleges through a formal, NAL-organized network."

The Report suggested that the landgrant and National Agricultural Library relationships fall into two categories: (1) the functional systems providing information for intermediate or ultimate users, and (2) the telecommunication system supportive of the information service functioning through rapid distribution of the services of the information network. It was advanced, furthermore, that the basic components providing the responses would be land-grant libraries, information analysis centers, and a telecommunication system.

In the system proposed, the sixty-nine land-grant institutions would be connected to a regional node by a narrow-band teletype line in a regional poll-and-select network. The control of each network would be exercised by a PABDX centrally located at the National Agricultural Library. Interconnection of each regional network within NAL would be by means of a narrow-band teletype line and voicegrade line. The regional nodes in the $\mathrm{Na}$ tional Agricultural Library would send messages to one another through a storeand-forward type of switching machine.

Included in the EDUCOM study was an investigation by the General Electric Company's Communications Products Department. Although the proposal of this group is only illustrative of a telecommunication system, it is an interesting feature of the Report. As a supplement to the basic document, it includes the rationale citing the assumptions, the system features, the explanations of the terminals as well as various estimates of costs for equipment and line charges. The GE group concluded that a well-designed communications network can be the difference between success and failure of a nationwide library network.

As the great thinker of modern librarianship, Verner Clapp, has recently said: "There is nothing new about library networks except the name." In considering networks we are in many ways really referring to the ancient concept of cooperation. In the land-grant institution tradition alone, we can look back as far as the Morrill Act in order to identify the genesis of our collaborative efforts in the sharing of information and resources.

What exactly then have the authors Becker, King, and Olsen proposed some 109 years later which is new and exciting? In the first place, they admit that their plan is a fundamental one and that its functions are not unique. Much of the report is, in fact, old hat in that it emphasizes the elements of interlibrary loan, photocopying service, referral centers, geographical depositories, and the coordination of acquisitions. These are not new concepts and they do not make the $R e$ port a landmark study of networks. In fairness to the team, on the other hand, this was not an objective of the EDUCOM investigation.

One missing element, nonetheless, is a sound reference to an acceptable philosophical position about the purpose of a library network. No matter how crude, no matter how sophisticated it is, this proposal can have meaning only in its relevance to the information which is provided with- 
in the network. In this instance, it is difficult to separate the agriculturalist's basic information need from that of any other scientist. If that is so, the advancement of this particular network only fragments further the larger information problem of control and dissemination.

Until a more coordinated national effort is offered, however, this proposed network is a forward step toward access to the totality of resources. At least with this kind of a system, we could have available a model to test involving the use of regional resources of a land-grant institution on a contractual basis. Whether such a network will result in an increase in selfsufficiency, a relaxation of demands upon the larger land-grant institutions, and a greater utilization of the National Agricultural Library remains to be seen.-LeMoyne W. Anderson, Colorado State University.

\section{Suppressed Commentaries on the Wiseian} Forgeries. Addendum to an Enquiry. By William B. Todd. Austin, Texas: Humanities Research Center, The University of Texas, 1969. 49p. (77-89555).

"Of making many books" we are told on good authority (Eccles. 12:12), "there is no end." So it is apparently going to be with the Wise saga. William Todd dedicates this Addendum, "To John Carter and Graham Pollard Whose Original Enquiry Will Lead to Endless Addenda on the Work of T. J. Wise."

There have already been a flood of books and articles on the subject since the Enquiry's original publication in 1934. An interesting aspect of this phenomenon is the fact that the book itself, though raising great interest in the book collecting world, and printed in a small edition, was a slow seller, taking perhaps two decades to go out of print; and it has not, to this day, seen a second edition.

William Todd here documents "four different campaigns undertaken by American nationals for or against the cause of $\mathrm{T}$. J. Wise," two by Charles Heartman, and the others by Gabriel Wells, both booksellers. In addition Heartman edited the American Book Collector and was a noted gadfly. Texas has acquired his files and corre- spondence about the magazine, from which Todd usefully reprints and annotates his correspondence with Wise, including a revealing article which Wise wrote (or had written for him) with the important sentences: "My own private opinion is that the Browning Sonnets is not genuine. The question is where did Mr. Forman obtain the 'Remainder' from." This article was never published, as Wise demanded its return, which he got, but not before Heartman retained some sort of copy. Miss Fannie Ratchford had seen this document, as Todd points out in a footnote, but failed to appreciate its significance.

Throughout the article Wise refers to Carter and Pollard as "the authors." In other correspondence I have seen, he called them "sewer rats."

The Gabriel Well's crusade (Todd calls it "folly") to vindicate Wise, which is documented, sprang from reasons not revealed in this Addendum.

The Ashley Library was the most spectacular one of the kind of books which were in fashion during the decades it was being formed and there was considerable speculation over its eventual disposal. Wells was, at that depression time, the most active dealer in Americana and the general opinion among his fellow dealers was that his defense of Wise was simply a ploy on his part to ingratiate himself with Mrs. Wise and others so he would be regarded favorably by them when and if an opportunity came along to purchase the collection. The Heartman correspondence with Wise is important to the saga, that of Wells is not.

Todd concludes with a "Postscript on The Story of a Lie" which documents Wise's methods of handling potentially dangerous problems, in this case, W. F. Prideaux's forthcoming bibliography of R. L. Stevenson.

The "Notes," of which there are fortyseven, are occasionally inadequate, or inaccurate, where contemporary personages are involved. Wilfred Partington is confused with (presumably) Henry M. Partridge. Certainly Mrs. Gertrude Hills and the equally formidable Leonard L. Mackall could be more satisfactorily identified, and Arthur Swann (of all people!) was 